\title{
ON THE SECOND HANKEL DETERMINANT OF AREALLY MEAN $p$-VALENT FUNCTIONS
}

BY

\author{
J. W. NOONAN( $\left.{ }^{1}\right)$ AND D. K. THOMAS
}

ABSTRACT. In this paper we determine the growth rate of the second Hankel determinant of an areally mean p-valent function. This result both extends and unifies previously known results concerning this problem.

I. Introduction and statement of results. Let $f$ be regular in $\gamma=\{z:|z|$ $<1\}$, with $f(z)=\sum_{n=0}^{\infty} a_{n} z^{n}$. The $q$ th Hankel determinant of $f$ is defined for $q \geqslant 1$ by

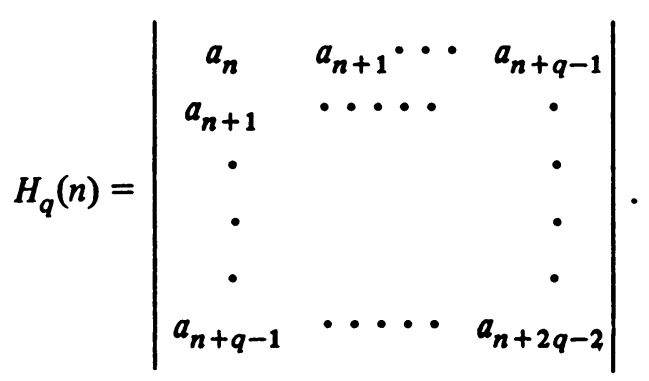

If $n(\omega)$ is the number of roots in $\gamma$ of the equation $f(z)=\omega, f$ is said to be areally mean $p$-valent in $\gamma$ [1] if for all $R>0$,

$$
W(R, f)=\frac{1}{\pi} \int_{0}^{2 \pi} \int_{0}^{R} n\left(\rho e^{i \theta}\right) \rho d \rho d \theta<p R^{2} .
$$

As usual, $f$ is normalized so that $\max \left\{\left|a_{k}\right|: 0 \leqslant k \leqslant[p]\right\}=1$, and the class of normalized areally mean $p$-valent functions is denoted by $S_{p}$.

The problem of determining the rate of growth of $H_{q}(n)$ as $n \rightarrow \infty$ when $f \in S_{p}$ is well known. Ch. Pommerenke [9] has shown that for $p \geqslant 1, H_{q}(n)=$ $O(1) n^{k \sqrt{q}-q / 2}$ where $k=16 p \sqrt{p}$. The present authors have shown [8] that if $q \geqslant 2$ and $p \geqslant 2(q-1)$, then $H_{q}(n)=O(1) n^{2 p q-q^{2}}$, where the exponent is best possible. For strictly univalent functions, Pommerenke [10] has shown that for $q \geqslant 2, H_{q}(n)=O(1) n^{-(1 / 2+\beta) q+3 / 2}$, where $\beta>1 / 4000$. In particular, $H_{2}(n)=O(1) n^{1 / 2-2 \beta}$. On the other hand, W. K. Hayman [4] has shown that $H_{2}(n)=o(1) n^{1 / 2}$ when $f \in S_{1}$, and that this is best possible.

It is clear that the known results concerning this problem are incomplete,

Received by the editors August 13, 1975.

AMS (MOS) subject classifications (1970). Primary 30A34.

( ${ }^{1}$ ) Supported by R.S.C. grant B/SR/22779.

Copyright $\odot$ 1976, American Muthematical Society 
in the sense that given $q$, best possible growth rates for $H_{q}(n)$ are known only for certain values of $p$. In this paper we shall examine the behavior of $\mathrm{H}_{2}(n)$ when $f \in S_{p}$. We prove

THEOREM 1. Let $f \in S_{p}$. Then, as $n \rightarrow \infty$,

$$
H_{2}(n)=a_{n} a_{n+2}-a_{n+1}^{2}= \begin{cases}o(1) n^{-1}, & 0<p<1 / 4, \\ o(1) n^{2 p-3 / 2}, & 1 / 4<p<5 / 4, \\ O(1) n^{4 p-4}, & p>5 / 4 .\end{cases}
$$

If $p>5 / 4$ and $\lim _{r \rightarrow 1}(1-r)^{2 p} M(r, f)=0$, then $H_{2}(n)=o(1) n^{4 p-4}$.

In the opposite direction we have

THEOREM 2. Given any positive sequence $\left\{\epsilon_{n}\right\}$ with $\lim _{n \rightarrow \infty} \epsilon_{n}=0$, there exists $f \in S_{p}$ such that for infinitely many $n$,

$$
\left|H_{2}(n)\right|> \begin{cases}\epsilon_{n} n^{-1}, & 0<p<1 / 4, \\ \epsilon_{n} n^{2 p-3 / 2}, & 1 / 4<p<5 / 4 .\end{cases}
$$

In addition, if $p>5 / 4$ and $f \in S_{p}$ satisfies $\alpha=\lim _{r \rightarrow 1}(1-r)^{2 p} M(r, f)>0$, then $\lim _{n \rightarrow \infty}\left|H_{2}(n)\right| / n^{4 p-4}=\alpha^{2}(2 p-1) / \Gamma(2 p)^{2}$.

Theorem 2 shows that the results of Theorem 1 are best possible, and also that when $p>5 / 4, O(1)$ cannot in general be replaced by $o(1)$. These results essentially solve Problem $6.14^{\prime}$ of [2] when $q=2$.

II. Preliminary results. With $f(z)=\Sigma_{n=0}^{\infty} a_{n} z^{n}$ and $y$ any complex number, we set $\Delta_{0}(n+2, y, f)=a_{n+2}, \Delta_{1}(n+1, y, f)=a_{n+1}-y a_{n+2}$, and $\Delta_{2}(n, y, f)=a_{n}-2 y a_{n+1}+y^{2} a_{n+2}$, so that

$$
H_{2}(n)=\Delta_{2}(n, y, f) \Delta_{0}(n+2, y, f)-\Delta_{1}(n+1, y, f)^{2} .
$$

We shall estimate the various terms in (2.1) by combining two methods due originally to W. K. Hayman [3], [4]. For the sake of brevity, we shall refer to the existing literature whenever possible.

If $z_{1} \in \gamma$ and $z=\rho e^{i \theta}$, Cauchy's theorem gives that

$$
\left|\Delta_{2}\left(n, z_{1}, z f^{\prime}\right)\right| \leqslant \frac{1}{2 \pi \rho^{n+1}} \int_{0}^{2 \pi}\left|z-z_{1}\right|^{2}\left|f^{\prime}(z)\right| d \theta,
$$

and upon integrating from $\rho=1-3 / n$ to $\rho=1-2 / n$, we find that

$$
\frac{\left|\Delta_{2}\left(n, z_{1}, z f^{\prime}\right)\right|}{n}=O(1) \int_{0}^{2 \pi} \int_{1-3 / n}^{1-2 / n}\left|z-z_{1}\right|^{2}\left|f^{\prime}(z)\right| \rho d \rho d \theta \text {. }
$$

Henceforth we assume that $n$ is fixed and that $z_{1}$ has been chosen so that 
$\left|z_{1}\right|=n /(n+1),\left|f\left(z_{1}\right)\right|=M(n /(n+1), f)$. We also set $M_{1}=\left|f\left(z_{1}\right)\right|, M_{k}=$ $e^{1-k} M_{1}, k \geqslant 1$.

Technical considerations dictate that we now proceed in two similar yet different ways. We first divide $E=\{z: 1-3 / n \leqslant \rho \leqslant 1-2 / n\}$ into disjoint subsets $E_{k}=\left\{z \in E: M_{k+1} \leqslant|f(z)|<M_{k}\right\}$. Upon using the techniques of [3] (see also $[8$, p. 508]) we conclude that

$$
\frac{\left|\Delta_{2}\left(n, z_{1}, z f^{\prime}\right)\right|}{n}=O(1) \sum_{k=1}^{\infty}\left\{\int_{0}^{2 \pi} \int_{1-3 / n}^{1-2 / n}\left|z-z_{1}\right|^{4} G_{k}(|f(z)|) \rho d \rho d \theta\right\}^{1 / 2},
$$

where $G_{k}(R)=M_{k}^{2} R^{2} /\left(M_{k}^{2}+R^{2}\right)$.

Following Hayman [4], we now introduce a slightly different method. Choosing $\lambda>2$, applying the Schwarz inequality to (2.2), and noting that

$$
\int_{0}^{2 \pi} \int_{1-3 / n}^{1-2 / n}\left|f^{\prime}(z)\right|^{2}\left(1+|f(z)|^{\lambda}\right)^{-1} \rho d \rho d \theta \leqslant A(\lambda)
$$

(see $[4$, p. 81$]$ ), we deduce that

$$
\begin{aligned}
& \frac{\left|\Delta_{2}\left(n, z_{1}, z f^{\prime}\right)\right|}{n} \\
& \quad \leqslant A(\lambda)\left\{n^{-1 / 2}+\left(\int_{0}^{2 \pi} \int_{1-3 / n}^{1-2 / n}\left|z-z_{1}\right|^{4}|f(z)|^{\lambda} \rho d \rho d \theta\right)^{1 / 2}\right\} .
\end{aligned}
$$

(As usual, $A_{1}, A_{2}, \ldots$ denote absolute constants, while $A(x, y, \ldots$ ) denotes a constant depending only on $x, y, \ldots$ )

The estimate (2.3) will be used when $p>5 / 4$, and (2.4) will be used when $1 / 4<p<5 / 4$.

III. Estimate for (2.4). Applying [6, Lemma 2] and [4, Lemma 3] to $\int_{0}^{2 \pi}\left|\rho e^{i \theta}-z_{1}\right|^{4}\left|f\left(\rho e^{i \theta}\right)\right|^{\lambda} d \theta$, we find that

$$
\int_{0}^{2 \pi}\left|\rho e^{i \theta}-z_{1}\right|^{4}\left|f\left(\rho e^{i \theta}\right)\right|^{\lambda} d \theta \leqslant A(p, \lambda)+A_{1}\left(J_{1}(\rho)+J_{2}(\rho)\right),
$$

where

$$
\begin{aligned}
& J_{1}(\rho)=\int_{1 / 2}^{\rho} \int_{0}^{2 \pi}\left|r e^{i \theta}-z_{1}\right|^{2}\left|f\left(r e^{i \theta}\right)\right|^{\lambda} r \log \rho / r d \theta d r, \\
& J_{2}(\rho)=\int_{1 / 2}^{\rho} \int_{0}^{2 \pi}\left|r e^{i \theta}-z_{1}\right|^{4}\left|f^{\prime}\left(r e^{i \theta}\right)\right|^{2}\left|f\left(r e^{i \theta}\right)\right|^{\lambda-2} r \log \rho / r d \theta d r .
\end{aligned}
$$

The essential part of our proof consists of deriving appropriate estimates for $J_{1}(\rho)$ and $J_{2}(\rho)$. We begin with $J_{2}(\rho)$. 
LEMma 1. Let $f \in S_{p}, \lambda>2, k$ a positive integer. Then for any a satisfying $0<a \leqslant k$, we have

$$
\begin{aligned}
\int_{1 / 2}^{\rho} \int_{0}^{2 \pi}\left|r e^{i \theta}-z_{1}\right|^{2 k}\left|f^{\prime}\left(r e^{i \theta}\right)\right|^{2}\left|f\left(r e^{i \theta}\right)\right|^{\lambda-2} r \log \rho \mid r d \theta d r & \left\{\begin{array}{l}
\left(\frac{n^{2 p}}{M_{1}}\right)^{a^{2} / 2 p} \text { if } 1<2 p \lambda<2 a+1, \\
\left(\frac{n^{2 p}}{M_{1}}\right)^{a^{2} / 2 p} \min \left\{M_{1},(1-\rho)^{-2 p}\right\}(2 p \lambda-2 a-1) / 2 p \\
\quad \text { if } 2 p \lambda>2 a+1 .
\end{array}\right.
\end{aligned}
$$

Proof. Divide the range of integration into subsets $F_{j}=\left\{r e^{i \theta}: 1 / 2 \leqslant\right.$ $\left.r<\rho, M_{j+1} \leqslant\left|f\left(r e^{i \theta}\right)\right|<M_{j}\right\}$; also note that $\log \rho / r<2(1-r)$. Following Hayman [4], we suppose that $M_{j+1} \geqslant M(1 / 2, f)$ for at least one value of $j$. (The opposite case is trivial.) Since $0<a \leqslant k,\left|r e^{i \theta}-z_{1}\right|^{2 k} \leqslant A\left|r e^{i \theta}-z_{1}\right|^{2 a}$, and so [5, Theorem 1]

$$
\left|r e^{i \theta}-z_{1}\right|^{2 k} \leqslant A(p, a)\left(n^{2 p} / M_{1}\right)^{a^{2} / 2 p}(1-r)^{-1}\left|f\left(r e^{i \theta}\right)\right|^{(-2 a-1) / 2 p} .
$$

Therefore

where $\epsilon=-\lambda+(2 a+1) / 2 p$.

$$
\begin{gathered}
\iint_{F_{j}}\left|r e^{i \theta}-z_{1}\right|^{2 k}\left|f^{\prime}\left(r e^{i \theta}\right)\right|^{2}\left|f\left(r e^{i \theta}\right)\right|^{\lambda-2} \log \rho / r d \theta d r \\
\leqslant A(p, a)\left(\frac{n^{2 p}}{M_{1}}\right)^{a^{2} / 2 p} M_{j}^{-\epsilon}
\end{gathered}
$$

If $\epsilon>0$ (i.e. $2 p \lambda<2 a+1$ ), we choose constants $b$ and $A(p)$ with $0<$ $b \leqslant M(1 / 2, f) \leqslant A(p)$, we define $j_{0}=j_{0}(n)=\max \left\{j: M_{j+1} \geqslant M(1 / 2, f)\right\}$, and we conclude that $b \leqslant M_{j_{0}+1} \leqslant A(p)$. With $F^{\prime \prime}=\left\{r e^{i \theta}:\left|f\left(r e^{i \theta}\right)\right| \leqslant M_{j_{0}+1}\right\}$, it follows easily from the definition of the class $S_{p}$ that

$$
\int_{F^{\prime \prime}}\left|r e^{i \theta}-z_{1}\right|^{2 k}\left|f^{\prime}\left(r e^{i \theta}\right)\right|^{2}\left|f\left(r e^{i \theta}\right)\right|^{\lambda-2} \log \rho / r d r d \theta \leqslant A(p, \lambda, k) .
$$

The above remarks therefore imply

$$
\begin{gathered}
\int_{1 / 2}^{\rho} \int_{0}^{2 \pi}\left|r e^{i \theta}-z_{1}\right|^{2 k}\left|f^{\prime}\left(r e^{i \theta}\right)\right|^{2}\left|f\left(r e^{i \theta}\right)\right|^{\lambda-2} r \log \rho / r d \theta d r \\
\quad=\iint_{F^{\prime \prime}}+\sum_{j=1}^{j_{0}} \int_{F_{j}} \\
\leqslant A(p, \lambda, k)+A(p, a)\left(\frac{n^{2 p}}{M_{1}}\right)^{a^{2} / 2 p} \sum_{j=1}^{j_{0}} M_{j}^{-\epsilon}
\end{gathered}
$$


Since $\epsilon>0, \Sigma_{j=1} M_{j}^{\epsilon} \leqslant b^{-\epsilon} \sum_{j=1}^{\infty} e^{-\epsilon}<\infty$, and the lemma follows.

If $\epsilon<0$ (i.e. $2 p \lambda>2 a+1$ ), we divide the range of integration into subsets $E_{j}=\left\{r e^{i \theta}: 1 / 2 \leqslant r<\rho, N_{j+1} \leqslant\left|f\left(r e^{i \theta}\right)\right|<N_{j}\right\}$, where $N_{1}=$ $\min \left\{M_{1}, A(p)(1-\rho)^{-2 p}\right\}, N_{j}=e^{1-j} N_{1}$. As above, we conclude that

$$
\begin{gathered}
\int_{E_{j}}\left|r e^{i \theta}-z_{1}\right|^{2 k}\left|f^{\prime}\left(r e^{i \theta}\right)\right|^{2}\left|f\left(r e^{i \theta}\right)\right|^{\lambda-2} \log \rho / r d r d \theta \\
\leqslant A(p, a, \lambda)\left(n^{2 p} / M_{1}\right)^{a^{2} / 2 p} N_{j}^{\epsilon} .
\end{gathered}
$$

Upon summing from $j=1$ to $\infty$ and using the fact that

$$
\sum_{j=1}^{\infty} N_{j}^{-\epsilon} \leqslant A(p, \lambda, a) N_{1}^{-\epsilon},
$$

we arrive at the conclusion of the lemma.

We now estimate $J_{1}(\rho)$.

Lemma 2. Let $f \in S_{p}, \lambda>2$, and $0<\alpha \leqslant 1$. Suppose that $1<2 p \lambda<$ $2 \alpha+3$ and $2 p \lambda \neq 2 \alpha+1,2 p \lambda \neq 3$. Then $J_{1}(p) \leqslant A(p, \alpha, \lambda)\left(n^{2 p} / M_{1}\right)^{\alpha^{2} / 2 p}$.

Proof. Using [6, Lemma 2] and [4, Lemma 3], we see that

where

$$
K(r) \leqslant A_{1}+16 K_{1}(r)+4 \lambda^{2} K_{2}(r)
$$

$$
\begin{aligned}
K(r) & =\int_{0}^{2 \pi}\left|r e^{i \theta}-z_{1}\right|^{2}\left|f\left(r e^{i \theta}\right)\right|^{\lambda} d \theta \\
K_{1}(r) & =\int_{1 / 2}^{r} \int_{0}^{2 \pi}\left|f\left(t e^{i \theta}\right)\right|^{\lambda}(1-t) d \theta d t \\
K_{2}(r) & =\int_{1 / 2}^{r} \int_{0}^{2 \pi}\left|t e^{i \theta}-z_{1}\right|^{2}\left|f\left(t e^{i \theta}\right)\right|^{\lambda-2}\left|f^{\prime}\left(t e^{i \theta}\right)\right|^{2}(1-t) d \theta d t .
\end{aligned}
$$

From Lemma 1 (with $k=1$ ), we find that

$$
K_{2}(r) \leqslant A(p, \lambda, \alpha)\left\{\begin{aligned}
\left(n^{2 p} / M_{1}\right)^{\alpha^{2} / 2 p}, & \text { if } 1<2 p \lambda<2 \alpha+1, \\
\left(n^{2 p} / M_{1}\right)^{\alpha^{2} / 2 p} \min \left\{M_{1},(1-r)^{-2 p}\right\}(2 p \lambda-2 \alpha-1) / 2 p & \text { if } 2 p \lambda>2 \alpha+1 .
\end{aligned}\right.
$$

Also, from [1, Theorem 3.2], we have $\int_{0}^{2 \pi}\left|f\left(t e^{i \theta}\right)\right|^{\lambda} d \theta \leqslant A(p, \lambda)(1-t)^{1-2 p \lambda}$, provided $2 p \lambda>1$. Thus

$$
K_{1}(r) \leqslant A(p, \lambda) \begin{cases}1, & \text { if } 1<2 p \lambda<3 \\ (1-r)^{3-2 p}, & \text { if } 2 p \lambda>3 .\end{cases}
$$

Hence 


$$
K(r) \leqslant A(p, \lambda, \alpha)\left(\frac{n^{2 p}}{M_{1}}\right)^{\alpha^{2} / 2 p} \begin{cases}1, & \text { if } 1<2 p \lambda<2 \alpha+1, \\ (1-r)^{1+2 \alpha-2 p \lambda,}, & \text { if } 2 \alpha+1<2 p \lambda<3 \\ & \text { or } 2 p \lambda>3 .\end{cases}
$$

Since $J_{1}(\rho)=\int_{1 / 2}^{\rho} K(r) \log \rho / r d r$, the lemina now follows immediately.

We can now estimate $\Delta_{2}\left(n, z_{1}, z f^{\prime}\right)$. Choose $a$ such that $0<a \leqslant 2$, and put $\alpha=a / 2$. Then Lemmas 1 and 2 imply that

$$
J_{1}(\rho)+J_{2}(\rho) \leqslant A(p, \lambda, a)\left(n^{2 p} / M_{1}\right)^{a^{2} / 2 p}
$$

provided $1<2 p \lambda<2 a+1,2 p \lambda \neq a+1,2 p \lambda \neq 3$. Upon combining (2.4), (3.1), and (3.2), we find that

$$
\begin{aligned}
\frac{\left|\Delta_{2}\left(n, z_{1}, z f^{\prime}\right)\right|}{n} & \leqslant A(\lambda)\left\{n^{-1 / 2}+A(p, a, \lambda)\left(\frac{n^{2 p}}{M_{1}}\right)^{a^{2} / 4 p}\left\{\int_{1-3 / n}^{1-2 / n} d \rho\right\}^{1 / 2}\right\} \\
& \leqslant A(p, a, \lambda) n^{-1 / 2}\left(\frac{n^{2 p}}{M_{1}}\right)^{a^{2} / 4 p}
\end{aligned}
$$

for any $a$ such that $0<a \leqslant 2,1<2 p \lambda<2 a+1,2 p \lambda \neq a+1,2 p \lambda \neq 3$.

IV. Proof of Theorem 1 when $0<p<1 / 4$ or $1 / 4<p<5 / 4$. If $0<p$ $<1 / 4$, then [11] $a_{n}=o(1) n^{-1 / 2}$, and so trivially $H_{2}(n)=o(1) n^{-1}$. Now suppose $1 / 4<p<5 / 4$. We first note that for $p>1 / 4$,

$$
\left|a_{n}\right| \leqslant A(p) n^{-1 / 2} M_{1}^{1-1 / 4 p}
$$

a result proved exactly as in [4] in the case $p=1$. Also, with $z_{1}=e^{i \theta} n_{n} /(n+1)$, we have

$$
\Delta_{2}\left(n, e^{i \theta} n, f\right)=n^{-1} \Delta_{2}\left(n, z_{1}, z f^{\prime}\right)+(n+1)^{-2} e^{2 i \theta} n_{n+2} .
$$

Combining this with (3.3), (4.1), and the fact that $1 / 4<p<5 / 4$, we see that

$$
\Delta_{2}\left(n, e^{i \theta} n, f\right) \leqslant A(p, a, \lambda) n^{-1 / 2}\left(n^{2 p} / M_{1}\right)^{a^{2} / 4 p}
$$

with $a$ as before.

We now prove that $H_{2}(n)=o(1) n^{2 p-3 / 2}$ when $1 / 4<p<5 / 4$. It follows from (4.1) and (4.2) that

$$
\Delta_{2}\left(n, e^{i \theta} n, f\right) \Delta_{0}\left(n+2, e^{i \theta} n, f\right) \leqslant A(p, a, \lambda) n^{2 p-3 / 2}\left(n^{2 p} / M_{1}\right)^{\delta},
$$

where $\delta=\left(a^{2}+1-4 p\right) / 4 p$. Choose $a=(2 p \lambda-1) / 2+\epsilon$, where $\lambda>2$ and $\epsilon>0$ are chosen such that all previous restrictions involving $a$ are satisfied, and also such that $\delta<0$. (Elementary computations verify that such a choice 
is possible; the fact that $1 / 4<p<5 / 4$ is essential here.)

We next note [6] that

$$
\left|\Delta_{1}\left(n+1, e^{i \theta} n, f\right)\right| \leqslant A(p, \lambda) \begin{cases}n^{2 p-2 \sqrt{p}}, & 1 / 4<p<1, \\ n^{2 p-2}, & p \geqslant 1,\end{cases}
$$

and so

$$
\left|\Delta_{1}\left(n+1, e^{i \theta} n, f\right)\right|^{2}=o(1) n^{2 p-3 / 2},
$$

where again we have used $1 / 4<p<5 / 4$. We thus conclude from (2.1) and the above remarks that

$$
\left|H_{2}(n)\right| / n^{2 p-3 / 2} \leqslant A(p)\left(M_{1} / n^{2 p}\right)^{-\delta}+o(1) .
$$

If $M_{1}=M(n /(n+1), f)=o(1) n^{2 p}$, then (since $\left.-\delta>0\right) H_{2}(n)=$ $o(1) n^{2 p-3 / 2}$. If $M_{1} \neq o(1) n^{2 p}$, it is well known [1] that $\lim _{r \rightarrow 1}(1-r)^{2 p} M(r, f)$ $>0$. From (3.1) and Lemmas 1 and 2, it follows that with $\lambda>2$ fixed, $\int_{0}^{2 \pi}\left|\rho e^{i \theta}-z_{1}\right|^{4}\left|f\left(\rho e^{i \theta}\right)\right|^{\lambda} d \theta$ is uniformly bounded for $0<\rho<1$. We now use exactly the same technique as does Hayman $[4$, p. 90] to conclude that $\Delta_{2}\left(n, z_{1}, z f^{\prime}\right)=o(1) n^{1 / 2}$, and then as above we deduce that

$$
H_{2}(n)=o(1) n^{2 p-3 / 2} \text {. }
$$

This completes the proof of Theorem 1 in the case $1 / 4<p<5 / 4$.

V. Estimate for (2.3). We now assume $p>5 / 4$. Our method is essentially that of [8], the major difference being that since we are dealing with the specific case $q=2, p>5 / 4$, we can make more efficient use of the two-point modulus bound than was possible in [8]. In view of the technical nature of this modification, we shall merely indicate the sort of changes to be made in [8]. Verification of the complete details will be left to the interested reader.

Recalling that $G_{k}(R)=M_{k}^{2} R^{2} /\left(M_{k}^{2}+R^{2}\right)$, we see upon using [6, Lemma 2] and [4, Lemma 3] that

$$
\int_{0}^{2 \pi}\left|\rho e^{i \theta}-z_{1}\right|^{4} G_{k}\left(\left|f\left(\rho e^{i \theta}\right)\right|\right) d \theta
$$

can be estimated in terms of seven integrals (see $[8$, p. 511]), of which the most troublesome is

$$
\int_{1 / 2}^{\rho}(1-t) \int_{0}^{2 \pi}\left|t e^{i \theta}-z_{1}\right|^{2} G_{k}\left(\left|f\left(t e^{i \theta}\right)\right|\right) d \theta t d t .
$$

Reapplying [6, Lemma 2] and [4, Lemma 3], we can estimate the inner integral of (5.1) in terms of seven more integrals, the most troublesome being

$$
\int_{1 / 2}^{t}\left(1-t_{1}\right) \int_{0}^{2 \pi}\left|t_{1} e^{i \theta}-z_{1}\right|^{2}\left|f^{\prime}\left(t_{1} e^{i \theta}\right)\right|^{2} \frac{M_{k}^{4}\left(M_{k}^{2}-R^{2}\right)}{\left(M_{k}^{2}+R^{2}\right)^{3}} d \theta t_{1} d t_{1}
$$


In order to estimate this integral we first replace the range of integration by $\Omega=\left\{t_{1} e^{i \theta}: 1 / 2 \leqslant t_{1}<t,\left|f\left(t_{1} e^{i \theta}\right)\right| \leqslant B_{1}\right\}$, where $B_{1}=$ $\min \left\{M_{k}, A(p)(1-t)^{-2 p}\right\}$. We now divide $\Omega$ into subsets $\Omega_{m}=\left\{t_{1} e^{i \theta} \in \Omega\right.$ : $\left.B_{m+1} \leqslant\left|f\left(t_{1} e^{i \theta}\right)\right|<B_{m}\right\}$, where $B_{m}=e^{1-m} B_{1}$. An application of the twopoint modulus bound (put $a=b=1$ in [5, Theorem 1]) allows us to conclude that integration over $\Omega_{m}$ contributes at most $A(p)\left(n^{2 p} / M_{1}\right)^{1 / 2 p} B_{m}^{(4 p-3) / 2 p}$, and upon summing from $m=1$ to $\infty$, we conclude that (5.2) is bounded above by $A(p)\left(n^{2 p} / M_{1}\right)^{1 / 2 p} \min \left\{M_{k}^{2-3 / 2 p},(1-t)^{3-4 p}\right\}$. Combining this estimate with the technique of $[8$, p. 517], we see that integration of $(5.2)$ contributes at most $A(p) e^{-(4 p-5) k / 2 p}\left(n^{2 p} / M_{1}\right)^{1 / 2 p} M_{1}^{(4 p-5) / 2 p}$ to (5.1).

After re-examining the arguments of [8] in light of the changes suggested above, we find that

$$
\int_{0}^{2 \pi}\left|\rho e^{i \theta}-z_{1}\right|^{4} G_{k}\left(\left|f\left(\rho e^{i \theta}\right)\right|\right) d \theta \leqslant A(p) e^{-(4 p-5) k / 4 p}\left(n^{2 p} / M_{1}\right)^{4 / 2} p_{n}^{4 p-5} .
$$

Summing these estimates (as required by (2.3)), we find that

$$
\frac{\left|\Delta_{2}\left(n, z_{1}, z f^{\prime}\right)\right|}{n} \leqslant A(p)\left(\frac{n^{2 p}}{M_{1}}\right)^{4 / 4 p} n^{2 p-3} .
$$

The important point concerning this method is that if $p>5 / 4$, the presence of the convergence factor $e^{-(4 p-5) k / 2 p}$ allows us to sum from $k=1$ to $\infty$ and obtain (5.3).

VI. Proof of Theorem 1 when $p>5 / 4$. The estimate (5.3) leads, in the same manner as in the case $1 / 4<p<5 / 4$, to the estimate

$$
\left|\Delta_{2}\left(n, e^{i \theta} n, f\right)\right| \leqslant A(p)\left(n^{2 p} / M_{1}\right)^{4 / 4 p} n^{2 p-3}
$$

Upon combining this with (4.1), we find that

$$
\left|\Delta_{2}\left(n, e^{i \theta} n, f\right) \Delta_{0}\left(n+2, e^{i \theta} n, f\right)\right| \leqslant A(p) n^{4 p-4}\left(M_{1} / n^{2 p}\right)^{4 p-5 / 4 p} .
$$

From (4.3) we have $\left|\Delta_{1}\left(n+1, e^{i \theta} n, f\right)\right|^{2} \leqslant A(p) n^{4 p-4}$, and upon combining this with (6.1) and (2.1), we conclude that $H_{2}(n)=O(1) n^{4 p-4}$, as required. (Here we have used the facts $p>5 / 4$ and $M_{1} \leqslant A(p) n^{2 p}$.)

If $M(r, f)=o(1)(1-r)^{-2 p}$, it is clear that $o(1)$ replaces $O(1)$ in (6.1), and from [7, Theorem 1] we have

$$
\Delta_{1}\left(n+1, e^{i \theta} n, f\right)=o(1) n^{2 p-2} \text { for } p>1 .
$$

Thus $H_{2}(n)=o(1) n^{4 p-4}$.

If $\alpha=\lim _{r \rightarrow 1}(1-r)^{2 p} M(r, f)>0$, it follows from [7, Theorem 4] that for $p>5 / 4$, 


$$
\lim _{n \rightarrow \infty} \frac{\left|H_{2}(n)\right|}{n^{4 p-4}}=\frac{\alpha^{2}(2 p-1)}{\Gamma(2 p)^{2}}
$$

Thus $O(1)$ cannot in general be replaced by $o(1)$ when $p>5 / 4$.

VII. Examples when $p<5 / 4$. Since (6.2) applies when $p>5 / 4$, we see that it remains only to prove the first statement of Theorem 2. Put $f_{0}(z)=$ $2^{2 p} \pi(1-z)^{-2 p}=\Sigma_{n=0}^{\infty} A_{n} z^{n}$ and $\varphi(z)=\Sigma_{n=2}^{\infty} b_{n} z^{n}$, where $\left\{b_{n}\right\}_{2}^{\infty}$ is any sequence of nonnegative numbers with $\Sigma_{n=2}^{\infty} b_{n} \leqslant 1, \Sigma_{n=2}^{\infty} n b_{n}^{2} \leqslant p$. In [7] it is shown that if $p \geqslant 1 / 2$, the function $f$ given by

$$
f(z)=f_{0}(z)+\varphi(z)=\sum_{n=0}^{\infty} a_{n} z^{n}
$$

is areally mean $p$-valent.

Suppose now that $\left\{\epsilon_{n}\right\}$ is as in Theorem 2, and choose $\left\{b_{n}\right\}$ such that for some subsequence $\left\{n_{k}\right\}, b_{n_{k}}=b_{n_{k}+2}=0, b_{n_{k}+1}=\epsilon_{n_{k}} n_{k}^{-1 / 2}$. Direct calculation, in which we use the fact that $a_{n}=A_{n}+b_{n}$, shows that

$$
H_{2}\left(n_{k}, f\right)=H_{2}\left(n_{k}, f_{0}\right)-2 A_{n_{k}+1} b_{n_{k}+1}-b_{n_{k}+1}^{2} \text {. }
$$

Since $\lim _{r \rightarrow 1}(1-r)^{2 p} M\left(r, f_{0}\right)>0$, it follows from (6.2) that

$$
\lim _{n_{k} \rightarrow \infty} \frac{H_{2}\left(n_{k}, f_{0}\right)}{n_{k}^{2 p-3 / 2}}=0
$$

where we have used strongly the fact that $p<5 / 4$. Also, $b_{n_{k}+1}^{2} / n_{k}^{2 p-3 / 2}=$ $\epsilon_{n_{k}}^{2} / n_{k}^{(4 p-1) / 2}$, and

$$
\frac{A_{n_{k}} b_{n_{k}+1}}{n_{k}^{2 p-3 / 2}}=\frac{2^{2 p} \pi \epsilon_{n_{k}}}{\Gamma(2 p)}(1+o(1)),
$$

since $A_{n} \sim 2^{2 p} \pi n^{2 p-1} / \Gamma(2 p)$. Theorem 2 (for $p \geqslant 1 / 2$ ) now follows immediately from these estimates and (7.1).

In order to prove Theorem 2 for $1 / 4<p<1 / 2$, we use the same technique as above, except that we are forced to alter the function $f$ slightly. Given $p$ with $1 / 4<p<1 / 2$, construct $F \in S_{p}$ as follows (see [3], [4]). Put $g(z)=(1-z)^{-1}$, $\chi=2 / \cos p \pi, G(z)=g(z)^{2 p}+\chi$, and $F(z)=G(z)+\varphi(z)$, where $\varphi$ is as before. Clearly all we need do to prove Theorem 2 is to show that $F \in S_{p}$.

Note first that $G$ maps $\gamma$ conformally into the sector

$$
E=\{\omega:|\arg (\omega-\chi)|<p \pi\} .
$$

Put $\omega=\chi+t e^{i \theta}$, so that $|\omega|^{2}=t^{2}+x^{2}+2 x t \cos \theta$. If $\omega \in E$, it follows from the definition of $\chi$ that $|\omega|^{2} \geqslant(t+2)^{2}$, and so $t \leqslant|\omega|-2$. 
Set $E_{R}=E \cap\{\omega:|\omega| \leqslant R\}$, and let $A(R)$ be the area of $E_{R}$. If $R<\chi$, then $A(R)=0$, while if $R>\chi, A(R)<p \pi(R-2)^{2}$. The argument employed by Hayman [3] now shows that $F \in S_{p}$. As noted above, this proves Theorem 2 for $1 / 4<p<1 / 2$. In conclusion, we note that the example given in $[1, \mathrm{p} .49]$ shows that Theorem 2 also holds for $0<p<1 / 4$.

\section{REFERENCES}

1. W. K. Hayman, Multivalent functions, Cambridge Tracts in Math. and Math. Phys., no. 48, Cambridge Univ. Press, Cambridge, 1958. MR 21 \#7302.

2. - Research problems in function theory, Athlone Press, London, 1967. MR 36 \#359.

3. - On successive coefficients of univalent functions, J. London Math. Soc. 38 (1963), 228-243. MR 26 \#6382.

4. - On the second Hankel determinant of mean univalent functions, Proc. London Math. Soc. (3) 18 (1968), 77-94. MR 36 \#2794.

5. K. W. Lucas, $A$ two-point modulus bound for areally mean p-valent functions, J. London Math. Soc. 43 (1968), 487-494. MR 37 \#1587.

6. - On successive coefficients of areally mean p-valent functions, J. London Math. Soc. 44 (1969), 631-642. MR 39 \#4379.

7. J. W. Noonan, Coefficient differences and Hankel determinants of areally mean pvalent functions, Proc. Amer. Math. Soc. 46 (1974), 29-37.

8. J. W. Noonan and D. K. Thomas, On the Hankel determinants of areally mean pvalent functions, Proc. London Math. Soc. (3) 25 (1972), 503-524. MR 46 \#5605.

9. Ch. Pommerenke, On the coefficients and Hankel determinants of univalent functions, J. London Math. Soc. 41 (1966), 111-122. MR 32 \#2575.

10. On the Hankel determinants of univalent functions, Mathematika 14 (1967), 108-112. MR 35 \#6811.

11. UUber die Mittelwerte und Koeffizienten multivalenter Funktionen, Math. Ann. 145 (1961/62), 285-296. MR 24 \#A3282.

\footnotetext{
DEPARTMENT OF MATHEMATICS, HOLY CROSS COLLEGE, WORCESTER, MASSACHUSETTS 01610
}

DEPARTMENT OF MATHEMATICS, UNIVERSITY COLLEGE OF SWANSEA, SWANSEA, GLAMORGAN, WALES 